\title{
An Impossibility Result for Linear Signal Processing Under Thresholding
}

\author{
Holger Boche, Senior Member, IEEE, and Ullrich J. Mönich, Student Member, IEEE
}

\begin{abstract}
In this paper, we analyze the approximation of the outputs of linear time-invariant systems by sampling series that use only the samples of the input signal. The samples are disturbed by the threshold operator, which sets all samples with an absolute value smaller than some threshold to zero. We do the analysis for the space of Paley-Wiener signals with absolutely integrable Fourier transform and show for the Hilbert transform that the peak approximation error can grow arbitrarily large for some signals in this space when the threshold approaches zero. This behavior is counterintuitive because one would expect a better behavior if the threshold was decreased. Since we consider oversampling and all kernels from a certain meaningful set, the results are valid not only for one specific approximation process, but for a whole class of approximation processes. Furthermore, we give a game theoretic interpretation of the problem in the setting of a game against nature and show that nature has a universal strategy to win this game.
\end{abstract}

Index Terms-Approximation, game against nature, Hilbert transform, Paley-Wiener space, sampling series, signal reconstruction.

\section{INTRODUCTION AND MOTIVATION}

$\mathbf{T}$ HE vast majority of physical phenomena and signal processing operations is linear. Linear problems have the advantage of being mathematically relatively easy to treat compared to nonlinear problems. However, not all phenomena and operations are linear. A frequent reason for nonlinearity in applications is the presence of a threshold operator.

One threshold operator is the operator that sets all signal values, whose absolute value is greater or equal to some threshold $\delta$, to $\delta$. This operator, which is often called the clipping operator, has been widely analyzed [1]-[3], because of its importance to many engineering applications, especially in power amplifier design. We do not address the clipping operator in this paper. Instead we do our analysis for the threshold operator that sets all signal values, whose absolute value is smaller than the threshold $\delta$, to zero, because it has several interesting applications and still there are few results available for it.

Wireless sensor networks are one possible application wherein the threshold operator is important. The sensors

Manuscript received September 05, 2008; accepted September 08, 2009. First published October 2, 2009; current version published February 10, 2010. This work was supported in part by the German Research Foundation (DFG) under Grant BO 1734/9-1. The associate editor coordinating the review of this manuscript and approving it for publication was Dr. Arie Yeredor.

The authors are with the Heinrich-Hertz-Chair for Mobile Communications, Technische Universität Berlin, D-10578 Berlin, Germany (e-mail: holger.boche@mk.tu-berlin.de; ullrich.moenich@mk.tu-berlin.de).

Digital Object Identifier 10.1109/TSP.2009.2033640 sample some bandlimited signal in space and time and transmit the samples to the receiving signal processing unit. In order to save energy, it is common to let the sensors transmit only if the absolute value of the signal exceeds some threshold $\delta>0$. Then, using these samples, the signal processing unit tries to approximate some transformation of the signal as good as possible. Although we use this sensor network scenario to motivate our analyses in this paper, the results are not limited to it and still valid in a more general, abstract setting.

We do not restrain ourselves to the analysis of one specific approximation process. Instead we treat the problem in a very general fashion by considering oversampling and all possible kernels in a meaningful class of reconstruction kernels.

A general approach to a problem is particularly useful when the problem under consideration is not well defined. An often encountered cause for this is the ambiguity in finding a suitable mathematical model for a complex nonmathematical problem. Using a general approach it is possible to solve a problem not only for one specific situation but for many different situations simultaneously. Apart from our approach to consider a whole class of approximation processes, axiomatic approaches are yet another possibility to achieve a high level of generality. Kuhn prizes the axiomatic approach in [4] by explicitly highlighting four game theoretical papers that use the axiomatic method. These are Milnor's paper "Games against nature" [5], Arrow's paper "A difficulty in the concept of social welfare" [6], where he introduced his "impossibility theorem," Shapley's paper "A value for n-person games" [7], and Nash's paper "The bargaining problem" [8]. We use the game against nature concept in this paper to give a game theoretic interpretation of our main result.

\section{BASIC PROBlem Formulation AND NotATION}

In order to continue, we need some notation and definitions. Let $\hat{f}$ denote the Fourier transform of a function $f$, where $\hat{f}$ is to be understood in the distributional sense. $L^{p}(\mathbb{R}), 1 \leq p<\infty$, is the space of all $p$ th-power Lebesgue integrable functions on $\mathbb{R}$, with the usual norm $\|\cdot\|_{p}$, and $L^{\infty}(\mathbb{R})$ is the space of all functions for which the essential supremum norm $\|\cdot\|_{\infty}$ is finite.

For $\sigma>0$, let $\mathcal{B}_{\sigma}$ be the set of all entire functions $f$ with the property that for all $\epsilon>0$ there exists a constant $C(\epsilon)$ with $|f(z)| \leq C(\epsilon) \exp ((\sigma+\epsilon)|z|)$ for all $z \in \mathbb{C}$. The Bernstein space $\mathcal{B}_{\sigma}^{p}$ consists of all signals in $\mathcal{B}_{\sigma}$, whose restriction to the real line is in $L^{p}(\mathbb{R}), 1 \leq p \leq \infty$. A signal in $\mathcal{B}_{\sigma}^{p}$ is called bandlimited to $\sigma$. By the Paley-Wiener-Schwartz theorem, the Fourier transform of a signal bandlimited to $\sigma$ is supported in $[-\sigma, \sigma]$. For $1 \leq p \leq 2$, the Fourier transform is defined in the classical and for $p>2$ in the distributional sense. It is well 
known that $\mathcal{B}_{\sigma}^{p} \subset \mathcal{B}_{\sigma}^{s}$ for $1 \leq p \leq s \leq \infty$. Hence, every signal $f \in \mathcal{B}_{\sigma}^{p}, 1 \leq p \leq \infty$, is bounded.

For $\sigma>0$ and $1 \leq p \leq \infty$, we denote by $\mathcal{P W}_{\sigma}^{p}$ the Paley-Wiener space of signals $f$ with a representation $f(z)=$ $1 /(2 \pi) \int_{-\sigma}^{\sigma} g(\omega) \mathrm{e}^{i z \omega} \mathrm{d} \omega, z \in \mathbb{C}$, for some $g \in L^{p}(-\sigma, \sigma)$. If $f \in \mathcal{P} \mathcal{W}_{\sigma}^{p}$, then $g(\omega)=\hat{f}(\omega)$. The norm for $\mathcal{P} \mathcal{W}_{\sigma}^{p}, 1 \leq p<$ $\infty$, is given by $\|f\|_{\mathcal{P} \mathcal{W}_{\sigma}^{p}}=\left(1 /(2 \pi) \int_{-\sigma}^{\sigma}|\hat{f}(\omega)|^{p} \mathrm{~d} \omega\right)^{1 / p}$. As a consequence of Parseval's equality, we have $\mathcal{B}_{\sigma}^{2}=\mathcal{P W}_{\sigma}^{2}$. Furthermore, the Hausdorff-Young inequality leads to $\mathcal{B}_{\sigma}^{q} \supset \mathcal{P} \mathcal{W}_{\sigma}^{p}$ for $1<p \leq 2,1 / p+1 / q=1$ and Hölder's inequality to

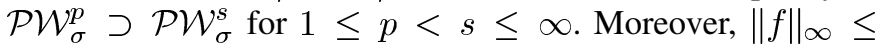
$\|f\|_{\mathcal{P} \mathcal{W}_{\sigma}^{1}}$.

\section{A. Signal Reconstruction Without and With Oversampling}

For signals in $\mathcal{P} \mathcal{W}_{\pi}^{1}$, the behavior of sampling series is often very different compared to their behavior for signals in $\mathcal{P} \mathcal{W}_{\pi}^{2}$. A well-known fact is the uniform convergence of the Shannon sampling series on compact subsets of $\mathbb{R}$ for all $f \in \mathcal{P} \mathcal{W}_{\pi}^{1}$ [9]-[11], [15]. That is, we have for all $f \in \mathcal{P} \mathcal{W}_{\pi}^{1}$ and $T>0$

$$
\lim _{N \rightarrow \infty} \max _{t \in[-T, T]}\left|f(t)-\sum_{k=-N}^{N} f(k) \frac{\sin (\pi(t-k))}{\pi(t-k)}\right|=0 .
$$

It would be of practical relevance to have the uniform convergence on all of $\mathbb{R}$. Then, it would be possible to bound the reconstruction error, which is made by the finite Shannon sampling series, on the whole real axis. Unfortunately, the Shannon sampling series is not uniformly convergent on all of $\mathbb{R}$ in general for $f \in \mathcal{P} \mathcal{W}_{\pi}^{1}$. Even worse, there exists a signal $f_{1} \in \mathcal{P} \mathcal{W}_{\pi}^{1}$, such that

$$
\limsup _{N \rightarrow \infty} \max _{t \in \mathbb{R}}\left|f(t)-\sum_{k=-N}^{N} f_{1}(k) \frac{\sin (\pi(t-k))}{\pi(t-k)}\right|=\infty
$$

i.e., the peak reconstruction error can grow arbitrarily large [12].

It is well known that oversampling improves the convergence behavior of the Shannon sampling series. Indeed, the application of oversampling leads to stable reconstruction processes and even the Shannon sampling series with the sinc-kernel and a slightly increased bandlimit is uniformly convergent on all of $\mathbb{R}$. For all $a>1$ and $f \in \mathcal{P} \mathcal{W}_{\pi}^{1}$, we have

$$
\lim _{N \rightarrow \infty} \max _{t \in \mathbb{R}}\left|f(t)-\sum_{k=-N}^{N} f\left(\frac{k}{a}\right) \frac{\sin \left(a \pi\left(t-\frac{k}{a}\right)\right)}{a \pi\left(t-\frac{k}{a}\right)}\right|=0 .
$$

Due to oversampling many different reconstruction kernels are possible, not only the sinc-kernel.

In particular, all kernels $\phi$ in $\mathcal{M}(a)$ can be used.

Definition 1: $\mathcal{M}(a), a>1$, is the set of functions $\phi \in \mathcal{B}_{a \pi}^{1}$ with $\hat{\phi}(\omega)=1 / a$ for $|\omega| \leq \pi$.

The functions in $\mathcal{M}(a), a>1$, are suitable kernels for the sampling series, because for all $f \in \mathcal{P} \mathcal{W}_{\pi}^{1}$ and $a>1$, we have

$$
\lim _{N \rightarrow \infty} \max _{t \in \mathbb{R}}\left|f(t)-\sum_{k=-N}^{N} f\left(\frac{k}{a}\right) \phi\left(t-\frac{k}{a}\right)\right|=0
$$

if $\phi \in \mathcal{M}(a)$.
Two well-known classes of kernels in $\mathcal{M}(a), a>1$, are the kernels with a trapezoidal shape in the frequency domain and the kernels with a cosine roll-off characteristic.

One important property of the kernels $\phi \in \mathcal{M}(a), a>1$, is stated in the following lemma.

Lemma 1: For all $a>1$ and $\phi \in \mathcal{B}_{a \pi}^{1}$, there exists a constant $C_{1}$ such that $\sum_{k=-\infty}^{\infty}|\phi(t-k / a)| \leq C_{1}\|\phi\|_{\mathcal{B}_{a \pi}^{1}}$ for all $t \in \mathbb{R}$.

Proof: For $g$ with $\hat{g} \in C_{0}^{\infty}[-(a+1) \pi,(a+1) \pi]$ and $\hat{g}(\omega)=1,|\omega| \leq a \pi$, we have

$$
\phi(t)=\int_{-\infty}^{\infty} \phi(\tau) g(t-\tau) \mathrm{d} \tau
$$

and

$$
\sum_{k=-N}^{N}\left|\phi\left(t-\frac{k}{a}\right)\right| \leq \int_{-\infty}^{\infty}|\phi(\tau)| \sum_{k=-N}^{N}\left|g\left(t-\frac{k}{a}-\tau\right)\right| \mathrm{d} \tau .
$$

Furthermore, since $\sum_{k=-N}^{N}|g(t-k / a-\tau)| \leq C_{1}$ it follows:

$$
\sum_{k=-\infty}^{\infty}\left|\phi\left(t-\frac{k}{a}\right)\right| \leq C_{1}\|\phi\|_{\mathcal{B}_{a \pi}^{1}} .
$$

The class of kernels $\mathcal{M}(a)$ is very important in the theory of systems, because, for all $\phi \in \mathcal{M}(a)$, the sampling series

$$
\left(A^{\phi} f\right)(t)=\sum_{k=-\infty}^{\infty} f\left(\frac{k}{a}\right) \phi\left(t-\frac{k}{a}\right)
$$

is bounded-input-bounded-output (BIBO) stable, which means that any bounded input signal $f$ leads to a bounded output signal $A^{\phi} f$.

By considering all kernels from $\mathcal{M}(a)$ in our analysis, the obtained results are valid not only for one specific reconstruction process but for a whole class of reconstruction processes.

\section{B. Thresholding Without and With Oversampling}

In Section II-A, we have seen that the peak reconstruction error can grow arbitrarily large, when using the Shannon sampling series without oversampling as reconstruction process. However, if certain operators are applied on the samples of the signal before the reconstruction, the situation might be different. Before we extend our discussion to the case where the samples are disturbed by the nonlinear threshold operator, we precisely introduce the threshold operator. For complex numbers $z \in \mathbb{C}$, the threshold operator $\kappa_{\delta}, \delta>0$, is defined by

$$
\kappa_{\delta} z= \begin{cases}z, & |z| \geq \delta \\ 0, & |z|<\delta\end{cases}
$$

Furthermore, for continuous signals $f: \mathbb{R} \rightarrow \mathbb{C}$, we define the threshold operator $\Theta_{\delta}, \delta>0$, pointwise, i.e., $\left(\Theta_{\delta} f\right)(t)=$ $\kappa_{\delta} f(t), t \in \mathbb{R}$.

In this paper, the threshold operator $\kappa_{\delta}$ is applied on the samples $\{f(k)\}_{k \in \mathbb{Z}}$ of signals $f \in \mathcal{P} \mathcal{W}_{\pi}^{1}$, which gives the disturbed samples $\left\{\kappa_{\delta} f(k)\right\}_{k \in \mathbb{Z}}$. This is, of course, equivalent to applying the threshold operator $\Theta_{\delta}$ on the signal $f$ itself and then taking the samples, i.e., $\left\{\left(\Theta_{\delta} f\right)(k)\right\}_{k \in \mathbb{Z}}$. 
The resulting samples $\left\{\left(\Theta_{\delta} f\right)(k)\right\}_{k \in \mathbb{Z}}, \delta>0$, can be used to build the approximation

$$
\begin{aligned}
\left(A_{\delta} f\right)(t) & :=\sum_{\substack{k=-\infty \\
|f(k)| \geq \delta}}^{\infty} f(k) \frac{\sin (\pi(t-k))}{\pi(t-k)} \\
& =\sum_{k=-\infty}^{\infty}\left(\Theta_{\delta} f\right)(k) \frac{\sin (\pi(t-k))}{\pi(t-k)}
\end{aligned}
$$

of the original signal $f$. The reconstruction process (1) uses only the samples that are larger or equal to the threshold $\delta$, and since the samples are taken at Nyquist rate, no other kernel than the sinc-kernel can be used. By $A_{\delta}$, we denote the operator that maps $f \in \mathcal{P} \mathcal{W}_{\pi}^{1}$ to $A_{\delta} f$ according to (1). Since $f \in \mathcal{P} \mathcal{W}_{\pi}^{1}$, we have $\lim _{t \rightarrow \infty} f(t)=0$ by the Riemann-Lebesgue lemma, and it follows that the series in (1) has only finitely many summands, which implies $A_{\delta} f \in \mathcal{P} \mathcal{W}_{\pi}^{2} \subset \mathcal{P} \mathcal{W}_{\pi}^{1}$.

In general, $A_{\delta} f$ is only an approximation of $f$, and we want the reconstructed signal $A_{\delta} f$ to be close to $f$ if $\delta$ is sufficiently small. We use the peak reconstruction error

$$
\left\|f-A_{\delta} f\right\|_{\infty}
$$

to measure this closeness. Since all signals $f \in \mathcal{P} \mathcal{W}_{\pi}^{1}$ are uniquely determined by their samples, and the series $A_{\delta} f, \delta>$ 0 , uses all "important" samples of the signal, i.e., all samples that are larger or equal than $\delta$, one could expect $A_{\delta} f$ to be a good approximation for $f$, at least if $\delta$ is small. However, this is not the case, because there exists a signal $f_{1} \in \mathcal{P} \mathcal{W}_{\pi}^{1}$ such that

$$
\limsup _{\delta \rightarrow 0}\left\|f_{1}-A_{\delta} f_{1}\right\|_{\infty}=\infty .
$$

Hence, a reduction of the threshold $\delta$ leads to an unbounded increase of the peak reconstruction error for some signals in $\mathcal{P W}_{\pi}^{1}$. This behavior is counterintuitive, because one would suspect that the reconstruction behavior of (1) gets better as the threshold $\delta$ is reduced.

Next we take a look on what happens in the case of thresholding and oversampling, i.e., we consider $a>1$. Using kernels $\phi \in \mathcal{M}(a)$, the reconstruction process with threshold has the shape

$$
\begin{aligned}
\left(A_{\delta}^{\phi} f\right)(t) & :=\sum_{\substack{k=-\infty \\
|f(k)| \geq \delta}}^{\infty} f\left(\frac{k}{a}\right) \phi\left(t-\frac{k}{a}\right) \\
& =\sum_{k=-\infty}^{\infty}\left(\Theta_{\delta} f\right)\left(\frac{k}{a}\right) \phi\left(t-\frac{k}{a}\right) .
\end{aligned}
$$

Again, as in the case without oversampling, for $f \in \mathcal{P} \mathcal{W}_{\pi}^{1}$, the series (4) has only finitely many samples, which implies $A_{\delta}^{\phi} f \in \mathcal{P W}_{a \pi}^{2} \subset \mathcal{P} \mathcal{W}_{a \pi}^{1}$. In contrast to reconstruction without oversampling, we now have a good approximation behavior of the reconstruction process (4). In [13], it has been shown that, for all $a>1, \phi \in \mathcal{M}(a)$, and $f \in \mathcal{P} \mathcal{W}_{\pi}^{1}$, we have

$$
\lim _{\delta \rightarrow 0}\left\|f-A_{\delta}^{\phi} f\right\|_{\infty}=0 .
$$

Equations (3) and (5) show that $A_{\delta}^{\phi} f$ exhibits a good approximation behavior for all $f \in \mathcal{P} \mathcal{W}_{\pi}^{1}$ as $\delta$ goes to zero only if oversampling is used.

\section{Thresholding and Stable LTI Systems}

However, in many signal processing applications, the task is to approximate some processed version $T f$ of $f \in \mathcal{P} \mathcal{W}_{\pi}^{1}$ and not $f$ itself. One frequently used type of processing is the filtering of a signal by a stable linear time-invariant (LTI) system $T$. So, another problem with an even higher practical relevance than the mere signal reconstruction is the approximation of $T f$ and therefore the behavior of

$$
\left\|T f-T A_{\delta}^{\phi} f\right\|_{\infty}
$$

for stable LTI systems $T: \mathcal{P} \mathcal{W}_{\pi}^{1} \rightarrow \mathcal{P} \mathcal{W}_{\pi}^{1}$ and $f \in \mathcal{P} \mathcal{W}_{\pi}^{1}$. As before, $\delta>0$ is the threshold and $A_{\delta}^{\phi}$ is the operator, defined in (4).

Before we continue the discussion, we briefly review some definitions and facts about stable LTI systems and introduce the Hilbert transform. A linear system $T: \mathcal{P W}_{\pi}^{1} \rightarrow \mathcal{P W}_{\pi}^{1}$ is called stable if the operator $T$ is bounded, i.e., if $\|T\|=$ $\sup _{\|f\|_{\mathcal{P W}}^{1} \leq 1}\|T f\|_{\mathcal{P} \mathcal{W}_{\pi}^{1}}<\infty$. Furthermore, it is called time invariant if $(T f(\cdot-a))(t)=(T f)(t-a)$ for all $f \in \mathcal{P} \mathcal{W}_{\pi}^{1}$ and $t, a \in \mathbb{R}$.

Note that our definition of stability is with respect to the $\mathcal{P W}_{\pi}^{1}$-norm and thus is different from the concept of BIBO stability. Our definition of stability is equivalent to the concept of energy stability, i.e., stability with respect to the $\mathcal{P} \mathcal{W}_{\pi}^{2}$-norm.

The Hilbert transform is one example of a stable LTI system, which has many applications [14]. The Hilbert transform $H f$ of a signal $f$ is defined by

$$
(H f)(t):=\frac{1}{2 \pi} \int_{-\infty}^{\infty}(-i \operatorname{sgn}(\omega)) \hat{f}(\omega) \mathrm{e}^{i \omega t} \mathrm{~d} \omega
$$

where sgn denotes the signum function. Note that the Hilbert transform is stable with respect to our definition of stability, but not BIBO stable. In Theorem 1, we use the Hilbert transform as one specific stable LTI system, however, the result is not restricted to the Hilbert transform, but also valid for other LTI systems that are stable with respect to our definition above.

\section{InTERPRETATION AS A GAME Against NATURE}

The problem, which is analyzed in this paper, is depicted in Fig. 1. Suppose that, according to the specific problem at hand, some arbitrary signal $f$ from the signal space $\mathcal{P} \mathcal{W}_{\pi}^{1}$ is given. In our wireless sensor network setting, this signal could be some physical quantity. By our measuring procedure, this signal is sampled in space with some oversampling factor $a>1$, and all samples with an absolute value below the threshold $\delta$ are set to zero. Next, the resulting samples $\left\{\left(\Theta_{\delta} f\right)(k / a)\right\}_{k \in \mathbb{Z}}$ are transmitted to the signal processing unit. In practical applications, we always have to deal with transmissions errors, which can lead to missing or erroneous samples at the receiver. For simplicity, we do not treat those problems and assume that the transmission is 


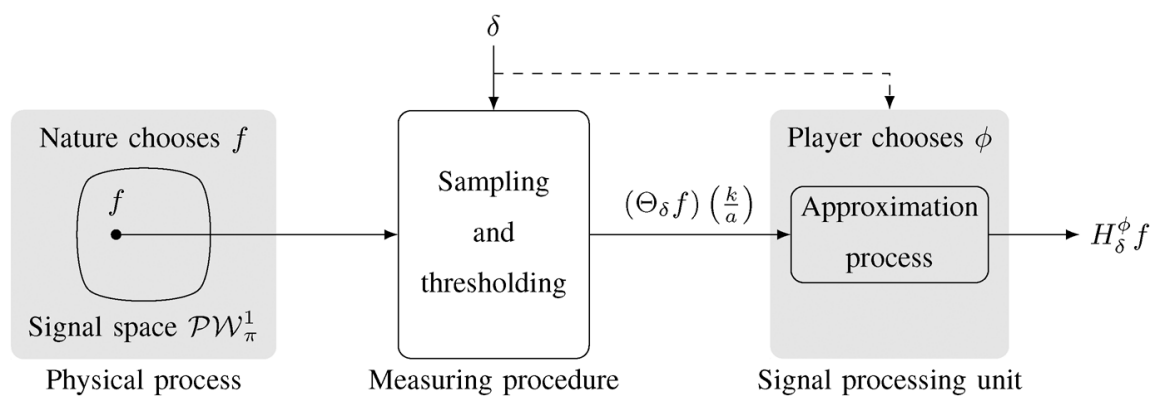

Fig. 1. Model of the game against nature.

error free. The signal processing unit uses the received samples to compute an approximation

$$
\begin{aligned}
H_{\delta}^{\phi} f & :=\sum_{\substack{k=-\infty \\
|f(k / a)| \geq \delta}}^{\infty} f\left(\frac{k}{a}\right)(H \phi)\left(t-\frac{k}{a}\right) \\
& =\sum_{k=-\infty}^{\infty}\left(\Theta_{\delta} f\right)\left(\frac{k}{a}\right)(H \phi)\left(t-\frac{k}{a}\right)
\end{aligned}
$$

of $H f$. As can be easily seen from (7), by the choice of $\phi \in$ $\mathcal{M}(a)$, we have a degree of freedom, which we can use to adapt the approximation process such that the approximation is as good as possible. For practical applications, we have to control the peak approximation error

$$
\left\|H f-H_{\delta}^{\phi} f\right\|_{\infty}
$$

for all possible thresholds $\delta>0$, i.e.,

$$
\sup _{0<\delta \leq 1}\left\|H f-H_{\delta}^{\phi} f\right\|_{\infty}
$$

has to be bounded. Intuitively one could suppose that the approximation error is reduced if the threshold is decreased. However, this is, as we will see in Theorem 1, not true in general.

In order to make the problem well posed, we have to bound the norm of the signals $f \in \mathcal{P} \mathcal{W}_{\pi}^{1}$. Otherwise, a simple scaling of $f$ could increase (8) unboundedly. We consider the set $\mathcal{N}=$ $\left\{f \in \mathcal{P} \mathcal{W}_{\pi}^{1}:\|f\|_{\mathcal{P W}} \leq 1\right\}$. The choice to upper bound $\|f\|_{\mathcal{P W}_{\pi}^{1}}$ by one in the definition of the set $\mathcal{N}$ is somehow arbitrary and only motivated by our special construction of the signal $f_{1}$ in the proof of Theorem 1 .

The whole problem can be interpreted as a game, played between nature and some intelligence, called player in the following. In this game, nature can choose an arbitrary signal $f \in$ $\mathcal{N}$ and the player can choose an arbitrary kernel $\phi \in \mathcal{M}(a)$ for the approximation process. The oversampling factor $a>1$ is supposed to be fixed in this game. Ideally, the player's goal would be to choose $\phi \in \mathcal{M}(a)$ so that (8) is minimized. However, the player has no direct access to the signal $f$; he knows only the sequence of disturbed samples $\left\{\left(\Theta_{\delta} f\right)(k / a)\right\}_{k \in \mathbb{Z}}$. So all he can do is to minimize

$$
\begin{aligned}
\sup _{0<\delta \leq 1}\left\|H_{\delta}^{\phi} f\right\|_{\infty} & \\
= & \sup _{0<\delta \leq 1}\left\|\sum_{k=-\infty}^{\infty}\left(\Theta_{\delta} f\right)\left(\frac{k}{a}\right)(H \phi)\left(\cdot-\frac{k}{a}\right)\right\|_{\infty} .
\end{aligned}
$$

In contrast, nature tries to maximize (9) by its choice of $f \in \mathcal{N}$. Finally, the player has won the game if $\sup _{0<\delta<1}\left\|H_{\delta}^{\phi} f\right\|_{\infty}<$ $\infty$, i.e., if the approximation error is bounded, and nature has won the game if $\sup _{0<\delta<1}\left\|H_{\delta}^{\phi} f\right\|_{\infty}=\infty$.

If $f \in \mathcal{N}$, we have $\|\bar{H} f\|_{\infty} \leq\|H f\|_{\mathcal{P} \mathcal{W}_{\pi}^{1}} \leq\|f\|_{\mathcal{P W}_{\pi}^{1}} \leq 1$ and consequently

$$
\begin{aligned}
\sup _{0<\delta \leq 1}\left\|H_{\delta}^{\phi} f\right\|_{\infty}-1 & \leq \sup _{0<\delta \leq 1}\left\|H f-H_{\delta}^{\phi} f\right\|_{\infty} \\
& \leq \sup _{0<\delta \leq 1}\left\|H_{\delta}^{\phi} f\right\|_{\infty}+1
\end{aligned}
$$

by applying the triangle inequality. Therefore, it is enough to control (9), because (10) automatically gives an upper bound on (8). In contrast, if nature has a strategy that increases (9) unboundedly, then the supremum of the approximation error (8) grows also arbitrarily large.

In Fig. 2, the game is illustrated as a matrix game. Note that the player's action space $\mathcal{N}$ and nature's action space $\mathcal{M}(a)$ are both uncountable. Thus, the interpretation of the game as a matrix game is only for illustrative purposes and not based on a mathematical justification.

The game can be played in two variants. In the fist variant, the player chooses $\phi$ without any additional knowledge. In the second variant, the player chooses $\phi$ with full knowledge about the sequence of disturbed samples $\left\{\left(\Theta_{\delta} f\right)(k / a)\right\}_{k \in \mathbb{Z}}$. The first situation might occur either if the player potentially has access to the information about the samples but decides, due to whatever reason, not to use it, or if it is impossible for the player to get the information because he has to make his choice of $\phi$ before nature chooses $f$. The second variant is typical for the situation where nature chooses first. Of course, the second variant is advantageous for the player because he has additional information in form of the sequence of disturbed samples, which he can use to adapt his choice of $\phi$ to nature's choice of $f$. Note that the player can neither deduce the threshold $\delta$ nor the original signal $f$ from the sequence of disturbed samples, because of the threshold operator.

In the first variant of the game, where the player has no additional knowledge, the best performance he can achieve-under the assumption that nature plays optimally—is given by

$$
\bar{E}=\inf _{\phi \in \mathcal{M}(a)} \sup _{f \in \mathcal{N}} \sup _{0<\delta \leq 1}\left\|H_{\delta}^{\phi} f\right\|_{\infty} .
$$

The reason can be seen in Fig. 2. For every $\phi \in \mathcal{M}(a)$, i.e., every row that the player chooses, nature can choose the 
signal $f \in \mathcal{N}$, i.e., the column for which $\sup _{0<\delta<1}\left\|H_{\delta}^{\phi} f\right\|_{\infty}$ is maximized. So the best the player can do is to minimize $\sup _{f \in \mathcal{N}} \sup _{0<\delta \leq 1}\left\|H_{\delta}^{\phi} f\right\|_{\infty}$ by his choice of $\phi \in \mathcal{M}(a)$.

In the second variant of the game, the player can adapt his choice of $\phi$ to nature's choice of $f$, which leads to

$$
\underline{E}=\sup _{f \in \mathcal{N}} \inf _{\phi \in \mathcal{M}(a)} \sup _{0<\delta \leq 1}\left\|H_{\delta}^{\phi} f\right\|_{\infty}
$$

as the best possible performance-again assuming that nature plays optimal. As before, the reason can be seen in Fig. 2. For every $f \in \mathcal{N}$, i.e., every column that nature chooses, the player can choose the function $\phi \in \mathcal{M}(a)$, i.e., the row for which $\sup _{0<\delta \leq 1}\left\|H_{\delta}^{\phi} f\right\|_{\infty}$ is minimized. So the best nature can do is to maximize $\inf _{\phi \in \mathcal{M}(a)} \sup _{0<\delta \leq 1}\left\|H_{\delta}^{\phi} f\right\|_{\infty}$ by its choice of $f \in$ $\mathcal{N}$. Note that although the player has only access to the disturbed samples $\left\{\left(\Theta_{\delta} f\right)(k / a)\right\}_{k \in \mathbb{Z}}$, and not to the full signal $f$, it is possible to achieve $\underline{E}$, because the approximation process $H_{\delta}^{\phi} f$ only uses the disturbed samples, which are known to the player.

Remark 1: Since we deal with infinite-dimensional spaces $\mathcal{N}$ and $\mathcal{M}(a)$, it is not clear whether an $f \in \mathcal{N}$ and a $\phi \in \mathcal{M}(a)$ exist such that the supremum and infimum in (11) and (12) are actually attained. However, it turns out that this problem is not present in our case.

Obviously, from (11) and (12), we see that $\underline{E} \leq \bar{E}$. The potentially better performance in the second variant of the game comes with the price of higher complexity: For every $f \in \mathcal{N}$, a new kernel $\phi \in \mathcal{M}(a)$ has to be determined, whereas in the first variant, the player uses only one single kernel for all $f \in \mathcal{N}$.

We will see in Theorem 1 that nature has a universal strategy, which is independent of the player's choice of $\phi \in \mathcal{M}(a)$. This strategy is given by playing always one universal signal $f_{1} \in \mathcal{N}$. For this signal, we have

$$
\limsup _{\delta \rightarrow 0}\left\|H_{\delta}^{\phi} f_{1}\right\|_{\infty}=\infty
$$

for all $\phi \in \mathcal{M}(a)$, and hence $\underline{E}=\infty$ as well as $\bar{E}=\infty$. This shows that an adaptive choice of the kernel $\phi$ is useless.

In some applications, the threshold $\delta$ is known a priori. Then both the player and nature can adapt their strategy to the threshold $\delta$, and the corresponding expressions for the best performance in the first and second variants of the game become

$$
\bar{E}_{\delta}=\sup _{0<\delta \leq 1} \inf _{\phi \in \mathcal{M}(a)} \sup _{f \in \mathcal{N}}\left\|H_{\delta}^{\phi} f\right\|_{\infty}
$$

and

$$
\underline{E}_{\delta}=\sup _{0<\delta \leq 1} \sup _{f \in \mathcal{N}} \inf _{\phi \in \mathcal{M}(a)}\left\|H_{\delta}^{\phi} f\right\|_{\infty}
$$

respectively. However, as we will see in Corollary 1, there exists a signal $f_{1} \in \mathcal{P} \mathcal{W}_{\pi}^{1}$ such that and hence $\underline{E}_{\delta}=\infty$ as well as $\bar{E}_{\delta}=\infty$. Therefore, additional side information in form of the threshold $\delta$ is useless for the player.

\section{MAIN THEOREM}

In this section, we state and prove the main theorem. An interpretation of it in the form of a game against nature has already been given in the previous section.

Theorem 1: Let $a>1$ be arbitrary. There exists a universal signal $f_{1} \in \mathcal{P} \mathcal{W}_{\pi}^{1}$ with $\left\|f_{1}\right\|_{\mathcal{P W}_{\pi}^{1}}=1$ such that for all $\phi \in$ $\mathcal{M}(a)$

$$
\limsup _{\delta \rightarrow 0}\left\|H_{\delta}^{\phi} f_{1}\right\|_{\infty}=\infty
$$

and consequently

$$
\limsup _{\delta \rightarrow 0}\left\|H f_{1}-H_{\delta}^{\phi} f_{1}\right\|_{\infty}=\infty .
$$

Remark 2: Theorem 1 immediately points out a universal strategy for nature in the game. If nature chooses the signal $f_{1}$ the player has no chance to bound $\lim \sup _{\delta \rightarrow 0}\left\|H_{\delta}^{\phi} f_{1}\right\|_{\infty}$ by a suitable choice of $\phi \in \mathcal{M}(a)$. As for the discussion in Remark 1, this means that the situation where the supremum and infimum in the expressions (11) and (12) are not attained at some $f \in \mathcal{N}$ and $\phi \in \mathcal{M}(a)$ cannot occur in our scenario.

A direct consequence of the proof of Theorem 1 is the following corollary.

Corollary 1: Let $a>1$ be arbitrary. There exists a signal $f_{1} \in \mathcal{P} \mathcal{W}_{\pi}^{1}$ with $\left\|f_{1}\right\|_{\mathcal{P W}_{\pi}^{1}}=1$ such that

$$
\limsup _{\delta \rightarrow 0} \inf _{\phi \in \mathcal{M}(a)}\left\|H_{\delta}^{\phi} f_{1}\right\|_{\infty}=\infty
$$

and consequently

$$
\limsup _{\delta \rightarrow 0} \inf _{\phi \in \mathcal{M}(a)}\left\|H f_{1}-H_{\delta}^{\phi} f_{1}\right\|_{\infty}=\infty .
$$

The divergence results of Theorem 1 and Corollary 1 are also valid for other LTI systems that are stable with respect to the $\mathcal{P W}_{\pi}^{1}$-norm. For BIBO-stable LTI systems, i.e., for LTI systems with absolutely integrable impulse response, the divergence phenomena, which we have encountered in Theorem 1 and Corollary 1, cannot occur.

Before we can prove Theorem 1, we need an additional Lemma.

Lemma 2: For all $a>1, f \in \mathcal{P} \mathcal{W}_{\pi}^{1}, L \in \mathbb{N}$, and $t \geq L / a$, we have

$$
\sum_{|k|<L}\left|f\left(\frac{k}{a}\right) r\left(t-\frac{k}{a}\right)\right|<a^{2}\|f\|_{\infty}
$$

where

$$
r(t)=\frac{2}{\pi^{2} t^{2}}\left(\sin (\pi t)-\sin \left(\frac{\pi}{2} t\right)\right) .
$$

$$
\limsup _{\delta \rightarrow 0} \inf _{\phi \in \mathcal{M}(a)}\left\|H_{\delta}^{\phi} f_{1}\right\|_{\infty}=\infty
$$




\begin{tabular}{|c|c|c|c|c|c|}
\hline & & & Nature chooses & & \\
\hline & & $f_{1}$ & $f_{2}$ & $f_{3}$ & $\ldots$ \\
\hline$\ddot{w}$ & $\phi_{1}$ & $\sup _{0<\delta \leq 1}\left\|H_{\delta}^{\phi_{1}} f_{1}\right\|_{\infty}$ & $\sup _{0<\delta \leq 1}\left\|H_{\delta}^{\phi_{1}} f_{2}\right\|_{\infty}$ & $\sup _{0<\delta \leq 1}\left\|H_{\delta}^{\phi_{1}} f_{3}\right\|_{\infty}$ & \\
\hline $\begin{array}{l}\overline{0} \\
\overline{0} \\
0\end{array}$ & $\phi_{2}$ & $\sup _{0<\delta \leq 1}\left\|H_{\delta}^{\phi_{2}} f_{1}\right\|_{\infty}$ & $\sup _{0<\delta \leq 1}\left\|H_{\delta}^{\phi_{2}} f_{2}\right\|_{\infty}$ & $\sup _{0<\delta \leq 1}\left\|H_{\delta}^{\phi_{2}} f_{3}\right\|_{\infty}$ & \\
\hline 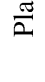 & $\phi_{3}$ & $\sup _{0<\delta \leq 1}\left\|H_{\delta}^{\phi_{3}} f_{1}\right\|_{\infty}$ & $\sup _{0<\delta \leq 1}\left\|H_{\delta}^{\phi_{3}} f_{2}\right\|_{\infty}$ & $\sup _{0<\delta \leq 1}\left\|H_{\delta}^{\phi_{3}} f_{3}\right\|_{\infty}$ & \\
\hline
\end{tabular}

Fig. 2. Infinite matrix game.

Proof: The simple sequence of inequalities

$$
\begin{aligned}
\sum_{|k|<L}\left|f\left(\frac{k}{a}\right) r\left(t-\frac{k}{a}\right)\right| & \leq\|f\|_{\infty} \sum_{|k|<L}\left|r\left(t-\frac{k}{a}\right)\right| \\
& \leq\|f\|_{\infty} \frac{4}{\pi^{2}} \sum_{|k|<L} \frac{1}{\left(t-\frac{k}{a}\right)^{2}} \\
& \leq\|f\|_{\infty} \frac{4 a^{2}}{\pi^{2}} \sum_{|k|<L} \frac{1}{(L-k)^{2}} \\
& <\|f\|_{\infty} \frac{4 a^{2}}{\pi^{2}} \sum_{k=1}^{\infty} \frac{1}{k^{2}} \\
& =\|f\|_{\infty} \frac{2 a^{2}}{3} \\
& <\|f\|_{\infty} a^{2}
\end{aligned}
$$

proves Lemma 2.

Now we are in the position to prove Theorem 1.

Proof of Theorem 1: Before we start with the proof, we shortly summarize the main steps. The central idea is to construct the desired signal $f_{1}$ as an infinite weighted sum of scaled and translated versions of one basic function. This basic function is the squared sinc function

$$
\left(\frac{\sin (\pi t)}{\pi t}\right)^{2}
$$

For the proof, it is essential that this function is positive. Let

$$
g_{M}(t)=\left(\frac{\sin \left(\frac{\pi t}{M}\right)}{\frac{\pi t}{M}}\right)^{2}
$$

$M \in \mathbb{N}$, denote the scaled version of (13). Two facts are important. First, the $\mathcal{P} \mathcal{W}_{\pi}^{1}$-norm $\left\|g_{M}\right\|_{\mathcal{P} \mathcal{W}_{\pi}^{1}}$ of $g_{M}$ is 1 , independently of $M \in \mathbb{N}$. Second, for every fixed $\delta$ with $1 / \pi^{2} \leq \delta<$ $1,\left\|H_{\delta}^{\phi} g_{M}\right\|_{\infty}$ goes to infinity as $M \rightarrow \infty$. By appropriately adding weighted and translated versions of $g_{M}$ with increasing $M$, we can construct a signal $f_{1}$ with $\|f\|_{\mathcal{P} \mathcal{W}_{\pi}^{1}}=1$ and a decreasing sequence $\left\{\delta_{l}\right\}_{l \in \mathbb{N}}$ such that $\lim _{l \rightarrow \infty}\left\|H_{\delta_{l}}^{\phi} f_{1}\right\|_{\infty}=\infty$. The construction of $f_{1}$ is done iteratively.

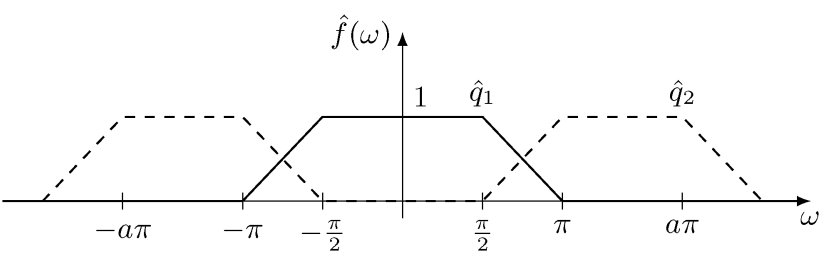

Fig. 3. Definition of $\hat{q}_{1}$ (solid line) and $\hat{q}_{2}$ (dashed line).

Now, we start with the proof. Let $a>1$ and be arbitrary but fixed. Furthermore, let $\hat{q}_{1}$ and $\hat{q}_{2}$ be the functions defined in Fig. 3 and $\phi \in \mathcal{M}(a)$ some arbitrary reconstruction kernel. Then, we have

$$
\phi=\phi * q_{1}+\phi * q_{2}=q_{1}+\phi * q_{2}
$$

and

$$
H \phi=H q_{1}+H\left(\phi * q_{2}\right)=H q_{1}+\underbrace{\phi *\left(H q_{2}\right)}_{=s} .
$$

Since $H q_{2} \in L^{1}(\mathbb{R})$, it follows that $s=\phi *\left(H q_{2}\right) \in L^{1}(\mathbb{R})$.

Moreover, for all $a>1, \phi \in \mathcal{M}(a), \delta>0$, and $f \in \mathcal{P} \mathcal{W}_{\pi}^{1}$, we have

$$
\begin{aligned}
\mid & \sum_{\substack{k=-\infty \\
|f(k / a)| \geq \delta}}^{\infty} f\left(\frac{k}{a}\right)(H \phi)\left(t-\frac{k}{a}\right) \\
& -\sum_{\substack{k=-\infty \\
|f(k / a)| \geq \delta}}^{\infty} f\left(\frac{k}{a}\right)\left(H q_{1}\right)\left(t-\frac{k}{a}\right) \mid \\
& =\left|\sum_{\substack{k=-\infty \\
|f(k / a)| \geq \delta}}^{\infty} f\left(\frac{k}{a}\right) s\left(t-\frac{k}{a}\right)\right| \\
\leq & \sum_{\substack{k=-\infty \\
|f(k / a)| \geq \delta}}^{\infty}\left|f\left(\frac{k}{a}\right)\right|\left|s\left(t-\frac{k}{a}\right)\right| \\
\leq & \|f\|_{\mathcal{P} \mathcal{W}_{\pi}^{1}} \sum_{k=-\infty}^{\infty}\left|s\left(t-\frac{k}{a}\right)\right| \\
\leq & C_{1}\|f\|_{\mathcal{P} \mathcal{W}_{\pi}^{1}}^{1}\|s\|_{\mathcal{B}}^{1}
\end{aligned}
$$


where we used Lemma 1 in the last inequality. Since we can upper bound the difference (14), it is enough to analyze

$$
\left(H_{\delta}^{q_{1}} f\right)(t):=\sum_{\substack{k=-\infty \\|f(k / a)| \geq \delta}}^{\infty} f\left(\frac{k}{a}\right)\left(H q_{1}\right)\left(t-\frac{k}{a}\right)
$$

in the following. For $t \neq 0$, we can simplify $\left(H q_{1}\right)(t)$, using integration by parts, according to

$$
\begin{aligned}
\left(H q_{1}\right)(t) & =\frac{1}{2 \pi} \int_{-\pi}^{\pi}-i \operatorname{sgn}(\omega) \hat{q}_{1}(\omega) \mathrm{e}^{i \omega t} \mathrm{~d} \omega \\
& =\frac{1}{\pi} \int_{0}^{\pi} \sin (\omega t) \hat{q}_{1}(\omega) \mathrm{d} \omega \\
& =\frac{1}{\pi t}-\underbrace{\frac{2}{\pi^{2} t^{2}}\left(\sin (\pi t)-\sin \left(\frac{\pi}{2} t\right)\right)}_{=r(t)}
\end{aligned}
$$

and for $t=0$, we have $\left(H q_{1}\right)(0)=0$.

Basic elements of the proof are the functions

$$
g_{M}(t)=\left(\frac{\sin \left(\frac{\pi t}{M}\right)}{\frac{\pi t}{M}}\right)^{2}, \quad M \in \mathbb{N}, \quad M \geq 2 .
$$

Every $g_{M}$ is the product of two sinc functions, and thus, in the frequency domain, $\hat{g}_{M}$ is the convolution of two rectangular functions, which implies $\hat{g}_{M}(\omega) \geq 0$ for all $\omega \in \mathbb{R}$. The nonnegativity of $\hat{g}_{M}$ can be used to calculate the $\mathcal{P} \mathcal{W}_{\pi}^{1}$-norm of $g_{M}$, because

$$
\begin{aligned}
\left\|g_{M}\right\|_{\mathcal{P} \mathcal{W}_{\pi}^{1}} & =\frac{1}{2 \pi} \int_{-\pi}^{\pi}\left|\hat{g}_{M}(\omega)\right| \mathrm{d} \omega=\frac{1}{2 \pi} \int_{-\pi}^{\pi} \hat{g}_{M}(\omega) \mathrm{d} \omega \\
& =g_{M}(0)=1
\end{aligned}
$$

for all $M \in \mathbb{N}, M \geq 2$. Since

$$
\left(\frac{\sin (\pi x)}{\pi x}\right)^{2}<\frac{1}{\pi^{2}}
$$

for all $|x| \geq 1$, we have $g_{M}(t)<1 / \pi^{2}$ for all $|t| \geq M$. For $0<\delta<1$ and $M \in \mathbb{N}$, let $k_{\delta}(M)$ denote the smallest natural number $k$ that fulfills

$$
g_{M}\left(\frac{k}{a}\right)=\left(\frac{\sin \left(\frac{\pi k}{a M}\right)}{\frac{\pi k}{a M}}\right)^{2}<\delta .
$$

Moreover, the function $((\sin (\pi x)) /(\pi x))^{2}$, which equals 1 for $x=0$ and 0 for $x=1$, is monotonically decreasing in $[0,1]$ and smaller than $1 / \pi^{2}$ for $|x| \geq 1$. Thus, for all $1 / \pi^{2} \leq \delta<1$, we know that the equation

$$
\left(\frac{\sin (\pi x)}{\pi x}\right)^{2}=\delta
$$

has a unique solution in the positive real numbers, which we will denote by $x_{\delta}$ in the following, and that $x_{\delta}$ satisfies $0<x_{\delta}<1$. Obviously

$$
\frac{k_{\delta}(M)}{a M}>x_{\delta}
$$

and

$$
k_{\delta}(M)>x_{\delta} a M .
$$

Next, we inductively construct a sequence $\left\{\psi_{k}\right\}_{k \in \mathbb{N}}$ of $\mathcal{P} \mathcal{W}_{\pi}^{1}$-functions. First, we set $\epsilon_{1}=1$ and choose some $\delta_{1}$ satisfying $\epsilon_{1} / \pi^{2}<\delta_{1}<\epsilon_{1}$. Then, we choose $M_{1} \in \mathbb{N}$, $M_{1} \geq 50$, so large that

$$
\frac{a \delta_{1}}{\pi} \log \left(x_{\eta_{1}} a M_{1}\right) \geq 2
$$

where $\eta_{1}=\delta_{1} / \epsilon_{1}=\delta_{1}$. The condition $M_{1} \geq 50$ is only a technical condition ensuring that $M_{1}$ is large enough such that $g_{M_{1}}(k / a) \leq g_{M_{1}}\left(k_{\delta_{1}}\left(M_{1}\right) / a\right)$ for all $k \geq \bar{k}_{1}$. For convenience, we introduce the abbreviation $\bar{k}_{1}=k_{\eta_{1}}\left(M_{1}\right)$. As the first function in the sequence, we set $\psi_{1}(t):=g_{M_{1}}(t)$.

Next, we need the constant

$$
C_{1}^{(1)}:=\frac{1}{2}\left(\delta_{1}-\psi_{1}\left(\frac{\bar{k}_{1}}{a}\right)\right)>0
$$

where the positivity of $C_{1}^{(1)}$ follows by the definition of $\bar{k}_{1}$. Now, we choose some $\epsilon_{2}$ satisfying

$$
0<\epsilon_{2} \leq \frac{1}{3} C_{1}^{(1)}
$$

and then some $\delta_{2}$ satisfying $\epsilon_{2} / \pi^{2}<\delta_{2}<\epsilon_{2}$. Furthermore, let $M_{2}>M_{1}$ be the smallest natural number such that

$$
\frac{a \delta_{2}}{\pi} \log \left(x_{\eta_{2}} a M_{2}\right) \geq 4
$$

where $\eta_{2}=\delta_{2} / \epsilon_{2}$. Again, we introduce the abbreviation $\bar{k}_{2}=k_{\eta_{2}}\left(M_{2}\right)$. As the second function in the sequence, we set $\psi_{2}(t)=\psi_{1}(t)+\epsilon_{2} g_{M_{2}}\left(t-T_{2} / a\right)$, where $T_{2}$ is the smallest natural number for which

$$
\left(\frac{a M_{1}}{\pi\left(T_{2}+\bar{k}_{2}\right)}\right)^{2}<\delta_{2}-\epsilon_{2} g_{M_{2}}\left(\frac{\bar{k}_{2}}{a}\right) .
$$

The first important property of $\psi_{2}$ is that for $|k| \geq \bar{k}_{1}$, we have

$$
\begin{aligned}
\psi_{2}\left(\frac{k}{a}\right) & =\psi_{1}\left(\frac{k}{a}\right)+\epsilon_{2} g_{M_{2}}\left(\frac{k-T_{2}}{a}\right) \\
& \leq \psi_{1}\left(\frac{k}{a}\right)+\epsilon_{2} \\
& \leq \psi_{1}\left(\frac{\bar{k}_{1}}{a}\right)+\epsilon_{2} \\
& <\delta_{1} .
\end{aligned}
$$

In the last inequality, we used (20) and (21). The second important property of $\psi_{2}$ is that for $|k| \geq \bar{k}_{2}+T_{2}$, we have

$$
\begin{aligned}
\psi_{2}\left(\frac{k}{a}\right) & =g_{M_{1}}\left(\frac{k}{a}\right)+\epsilon_{2} g_{M_{2}}\left(\frac{k-T_{2}}{a}\right) \\
& \leq\left(\frac{a M_{1}}{\pi k}\right)^{2}+\epsilon_{2} g_{M_{2}}\left(\frac{k-T_{2}}{a}\right) \\
& \leq\left(\frac{a M_{1}}{\pi\left(\bar{k}_{2}+T_{2}\right)}\right)^{2}+\epsilon_{2} g_{M_{2}}\left(\frac{\bar{k}_{2}}{a}\right) \\
& <\delta_{2}
\end{aligned}
$$


because of (23). A direct consequence of (24) is that

$$
\begin{aligned}
\left(H_{\delta_{1}}^{q_{1}} \psi_{2}\right)(t) & =\sum_{\substack{k=-\infty \\
\left|\psi_{2}(k / a)\right| \geq \delta_{1}}}^{\infty} \psi_{2}\left(\frac{k}{a}\right)\left(H q_{1}\right)\left(t-\frac{k}{a}\right) \\
& =\sum_{|k|<\bar{k}_{1}} \psi_{2}\left(\frac{k}{a}\right)\left(H q_{1}\right)\left(t-\frac{k}{a}\right) .
\end{aligned}
$$

Evaluating the expression (26) for $t=t_{1}^{*}=\left(\bar{k}_{1}+1\right) / a$ and inserting (15) leads to

$$
\begin{aligned}
\left(H_{\delta_{1}}^{q_{1}} \psi_{2}\right)\left(t_{1}^{*}\right)= & \sum_{|k|<\bar{k}_{1}} \psi_{2}\left(\frac{k}{a}\right) \frac{1}{\pi\left(\frac{\bar{k}_{1}+1}{a}-\frac{k}{a}\right)} \\
& -\sum_{|k|<\bar{k}_{1}} \psi_{2}\left(\frac{k}{a}\right) r\left(t_{1}^{*}-\frac{k}{a}\right) \\
\geq & \frac{a}{\pi} \sum_{|k|<\bar{k}_{1}} g_{M_{1}}\left(\frac{k}{a}\right) \frac{1}{\bar{k}_{1}+1-k} \\
& -\sum_{|k|<\bar{k}_{1}} \psi_{2}\left(\frac{k}{a}\right) r\left(t_{1}^{*}-\frac{k}{a}\right)
\end{aligned}
$$

where we used the fact that $g_{M_{2}}(t) \geq 0$ for all $t \in \mathbb{R}$. Since

$$
\left|\sum_{|k|<\bar{k}_{1}} \psi_{2}\left(\frac{k}{a}\right) r\left(t_{1}^{*}-\frac{k}{a}\right)\right|<\left(1+\epsilon_{2}\right) a^{2}
$$

by Lemma 2 , it follows that

$$
\begin{aligned}
\left(H_{\delta_{1}}^{q_{1}} \psi_{2}\right)\left(t_{1}^{*}\right) & \geq \frac{a \delta_{1}}{\pi} \sum_{|k|<\bar{k}_{1}} \frac{1}{\bar{k}_{1}+1-k}-\left(1+\epsilon_{2}\right) a^{2} \\
& >\frac{a \delta_{1}}{\pi} \log \left(\bar{k}_{1}\right)-\left(1+\epsilon_{2}\right) a^{2} \\
& \geq 2-\left(1+\epsilon_{2}\right) a^{2}
\end{aligned}
$$

where we used (19) in the last line. For $t_{2}^{*}=\left(\bar{k}_{2}+T_{2}+1\right) / a$, we have

$$
\begin{aligned}
\left(H_{\delta_{2}}^{q_{1}} \psi_{2}\right)\left(t_{2}^{*}\right)= & \sum_{\substack{k=-\infty \\
\left|\psi_{2}(k / a)\right| \geq \delta_{2}}}^{\infty} \psi_{2}\left(\frac{k}{a}\right)\left(H q_{1}\right)\left(t-\frac{k}{a}\right) \\
= & \sum_{\substack{k=-\infty \\
\left|\psi_{2}(k / a)\right| \geq \delta_{2}}}^{\infty} \psi_{2}\left(\frac{k}{a}\right) \frac{1}{\pi\left(t_{2}^{*}-\frac{k}{a}\right)} \\
& -\sum_{\substack{k=-\infty \\
\left|\psi_{2}(k / a)\right| \geq \delta_{2}}}^{\infty} \psi_{2}\left(\frac{k}{a}\right) r\left(t_{2}^{*}-\frac{k}{a}\right) .
\end{aligned}
$$

Since $\max \left\{k: \psi_{2}(k / a) \geq \delta_{2}\right\}<t_{2}^{*} a$ according to (25), every summand of the first sum on the right-hand side of (27) is pos- itive, and therefore, leaving out some summands only reduces the sum. Using $\epsilon_{2} g_{M_{2}}(t) \leq \psi_{2}(t), t \in \mathbb{R}$, we obtain

$$
\begin{aligned}
\left(H_{\delta_{2}}^{q_{1}} \psi_{2}\right)\left(t_{2}^{*}\right) \geq & \sum_{\left|k-T_{2}\right|<\bar{k}_{2}} \epsilon_{2} g_{M_{2}}\left(\frac{k}{a}\right) \frac{1}{\pi\left(t_{2}^{*}-\frac{k}{a}\right)} \\
& -\sum_{|k|<\bar{k}_{2}+T_{2}}\left|\psi_{2}\left(\frac{k}{a}\right) r\left(t_{2}^{*}-\frac{k}{a}\right)\right| \\
\geq & \frac{a \delta_{2}}{\pi} \sum_{\left|k-T_{2}\right|<\bar{k}_{2}}{\overline{k_{2}}+T_{2}+1-k}_{|k|<\bar{k}_{2}+T_{2}}\left|\psi_{2}\left(\frac{k}{a}\right) r\left(t_{2}^{*}-\frac{k}{a}\right)\right| .
\end{aligned}
$$

Since

$$
\sum_{|k|<\bar{k}_{2}+T_{2}}\left|\psi_{2}\left(\frac{k}{a}\right) r\left(t_{2}^{*}-\frac{k}{a}\right)\right|<\left(1+\epsilon_{2}\right) a^{2}
$$

by Lemma 2 , it follows that

$$
\begin{aligned}
\left(H_{\delta_{2}}^{q_{1}} \psi_{2}\right)\left(t_{2}^{*}\right) & >\frac{a \delta_{2}}{\pi} \sum_{\left|k-T_{2}\right|<\bar{k}_{2}} \frac{1}{\bar{k}_{2}+T_{2}+1-k}-\left(1+\epsilon_{2}\right) a^{2} \\
& \geq \frac{a \delta_{2}}{\pi} \log \left(\bar{k}_{2}\right)-\left(1+\epsilon_{2}\right) a^{2} \\
& \geq 4-\left(1+\epsilon_{2}\right) a^{2}
\end{aligned}
$$

where we used (22) in the last line.

Next, we need the constants

$$
C_{2}^{(1)}:=\frac{1}{2}\left(\delta_{2}-\psi_{2}\left(\frac{\bar{k}_{2}+T_{2}}{a}\right)\right)>0
$$

and

$$
C_{1}^{(2)}:=\frac{1}{2}\left(\delta_{1}-\psi_{1}\left(\frac{\bar{k}_{1}}{a}\right)-\epsilon_{2}\right)>0
$$

where the positivity of $C_{2}^{(1)}$ follows from (25). Now, we choose some $\epsilon_{3}$ satisfying

$$
0<\epsilon_{3} \leq \frac{1}{3} \min \left(C_{1}^{(2)}, C_{2}^{(1)}\right)
$$

and then some $\delta_{3}$ satisfying $\epsilon_{3} / \pi^{2}<\delta_{3}<\epsilon_{3}$. Furthermore, let $M_{3}>M_{2}$ be the smallest natural number such that

$$
\frac{a \delta_{3}}{\pi} \log \left(x_{\eta_{3}} a M_{3}\right) \geq 8
$$

where $\eta_{3}=\delta_{3} / \epsilon_{3}$. Again, we introduce the abbreviation $\bar{k}_{3}=$ $k_{\eta_{3}}\left(M_{3}\right)$. As the third function in the sequence, we set $\psi_{3}(t)=$ $\psi_{2}(t)+\epsilon_{3} g_{M_{3}}\left(t-T_{3} / a\right)$, where $T_{3}$ is the smallest natural number for which

$$
\left(\frac{a M_{1}}{\pi\left(\bar{k}_{3}+T_{3}\right)}\right)^{2}+\epsilon_{2}\left(\frac{a M_{2}}{\pi\left(\bar{k}_{3}+T_{3}-T_{2}\right)}\right)^{2}<\delta_{3}-\epsilon_{3} g_{M_{3}}\left(\frac{\bar{k}_{3}}{a}\right) \text {. }
$$


The first important property of $\psi_{3}$ is that for $|k| \geq \bar{k}_{1}$, we have

$$
\begin{aligned}
\psi_{3}\left(\frac{k}{a}\right) & =\psi_{2}\left(\frac{k}{a}\right)+\epsilon_{3} g_{M_{3}}\left(\frac{k-T_{3}}{a}\right) \\
& \leq \psi_{2}\left(\frac{k}{a}\right)+\epsilon_{3} \\
& \leq \psi_{1}\left(\frac{\bar{k}_{1}}{a}\right)+\epsilon_{2}+\epsilon_{3} \\
& <\delta_{1} .
\end{aligned}
$$

In the second to last inequality, we used (24), and in the last inequality, we used (29) and (30). A direct consequence of (33) is that for $t_{1}^{*}=\left(\bar{k}_{1}+1\right) / a$

$$
\begin{aligned}
\left(H_{\delta_{1}}^{q_{1}} \psi_{3}\right)\left(t_{1}^{*}\right)= & \sum_{\substack{k=-\infty \\
\left|\psi_{3}(k / a)\right| \geq \delta_{1}}}^{\infty} \psi_{3}\left(\frac{k}{a}\right)\left(H q_{1}\right)\left(t_{1}^{*}-\frac{k}{a}\right) \\
= & \sum_{|k|<\bar{k}_{1}} \psi_{3}\left(\frac{k}{a}\right)\left(H q_{1}\right)\left(t_{1}^{*}-\frac{k}{a}\right) \\
= & \sum_{|k|<\bar{k}_{1}} \psi_{3}\left(\frac{k}{a}\right) \frac{1}{\pi\left(\frac{\bar{k}_{1}+1}{a}-\frac{k}{a}\right)} \\
& -\sum_{|k|<\bar{k}_{1}} \psi_{3}\left(\frac{k}{a}\right) r\left(t_{1}^{*}-\frac{k}{a}\right) \\
\geq & \frac{a}{\pi} \sum_{|k|<\bar{k}_{1}} g_{M_{1}}\left(\frac{k}{a}\right) \overline{\bar{k}_{1}+1-k} \\
& -\sum_{|k|<\bar{k}_{1}} \psi_{3}\left(\frac{k}{a}\right) r\left(t_{1}^{*}-\frac{k}{a}\right)
\end{aligned}
$$

where we used the fact that $g_{M_{2}}(t) \geq 0$ and $g_{M_{3}}(t) \geq 0$ for all $t \in \mathbb{R}$. Since

$$
\left|\sum_{|k|<\bar{k}_{1}} \psi_{3}\left(\frac{k}{a}\right) r\left(t_{1}^{*}-\frac{k}{a}\right)\right|<\left(1+\epsilon_{2}+\epsilon_{3}\right) a^{2}
$$

by Lemma 2, it follows that

$$
\begin{aligned}
\left(H_{\delta_{1}}^{q_{1}} \psi_{3}\right)\left(t_{1}^{*}\right) & \geq \frac{a \delta_{1}}{\pi} \sum_{|k|<\bar{k}_{1}} \frac{1}{\bar{k}_{1}+1-k}-\left(1+\epsilon_{2}+\epsilon_{3}\right) a^{2} \\
& >\frac{a \delta_{1}}{\pi} \log \left(\bar{k}_{1}\right)-\left(1+\epsilon_{2}+\epsilon_{3}\right) a^{2} \\
& \geq 2-\left(1+\epsilon_{2}+\epsilon_{3}\right) a^{2}
\end{aligned}
$$

where we used (19) in the last line. The second important property of $\psi_{3}$ is that for $k \geq \bar{k}_{2}+T_{2}$, we have

$$
\begin{aligned}
\psi_{3}\left(\frac{k}{a}\right) & =\psi_{2}\left(\frac{k}{a}\right)+\epsilon_{3} g_{M_{3}}\left(\frac{k-T_{3}}{a}\right) \\
& \leq \psi_{2}\left(\frac{k}{a}\right)+\epsilon_{3} \\
& <\delta_{2}
\end{aligned}
$$

because of (28) and (30). Consequently, for $t_{2}^{*}=\left(\bar{k}_{2}+T_{2}+1\right) / a$

$$
\begin{aligned}
\left(H_{\delta_{2}}^{q_{1}} \psi_{3}\right)\left(t_{2}^{*}\right)= & \sum_{\substack{k=-\infty \\
\left|\psi_{3}(k / a)\right| \geq \delta_{2}}}^{\infty} \psi_{3}\left(\frac{k}{a}\right)\left(H q_{1}\right)\left(t_{2}^{*}-\frac{k}{a}\right) \\
= & \sum_{\substack{k=-\infty \\
\left|\psi_{3}(k / a)\right| \geq \delta_{2}}}^{\infty} \psi_{3}\left(\frac{k}{a}\right) \frac{1}{\pi\left(t_{2}^{*}-\frac{k}{a}\right)} \\
& -\sum_{\substack{k=-\infty \\
\left|\psi_{3}(k / a)\right| \geq \delta_{2}}}^{\infty} \psi_{3}\left(\frac{k}{a}\right) r\left(t_{2}^{*}-\frac{k}{a}\right) .
\end{aligned}
$$

Since $\max \left\{k: \psi_{3}(k / a) \geq \delta_{2}\right\}<t_{2}^{*} a$ according to (34), every summand of the first sum on the right-hand side of (35) is positive, and therefore, leaving out some summands only reduces the sum. Using Lemma 2, it follows that

$$
\begin{aligned}
\left(H_{\delta_{2}}^{q_{1}} \psi_{3}\right)\left(t_{2}^{*}\right) \geq & \sum_{\left|k-T_{2}\right|<\bar{k}_{2}} \epsilon_{2} g_{M_{2}}\left(\frac{k}{a}\right) \frac{1}{\pi\left(t_{2}^{*}-\frac{k}{a}\right)} \\
& -\sum_{|k|<\bar{k}_{2}+T_{2}}\left|\psi_{3}\left(\frac{k}{a}\right) r\left(t_{1}^{*}-\frac{k}{a}\right)\right| \\
& >\frac{a \delta_{2}}{\pi} \log \left(\bar{k}_{2}\right)-\left(1+\epsilon_{2}+\epsilon_{3}\right) a^{2} \\
& >4-\left(1+\epsilon_{2}+\epsilon_{3}\right) a^{2}
\end{aligned}
$$

by applying (22) in the last inequality. The third important property of $\psi_{3}$ is that for $|k| \geq \bar{k}_{3}+T_{3}$, we have

$$
\begin{aligned}
\psi_{3}\left(\frac{k}{a}\right)= & g_{M_{1}}\left(\frac{k}{a}\right)+\epsilon_{2} g_{M_{2}}\left(\frac{k-T_{2}}{a}\right)+\epsilon_{3} g_{M_{3}}\left(\frac{k-T_{3}}{a}\right) \\
\leq & \left(\frac{a M_{1}}{\pi k}\right)^{2}+\epsilon_{2}\left(\frac{a M_{2}}{\pi\left(k-T_{2}\right)}\right)^{2}+\epsilon_{3} g_{M_{3}}\left(\frac{k-T_{3}}{a}\right) \\
\leq & \left(\frac{a M_{1}}{\pi\left(\bar{k}_{3}+T_{3}\right)}\right)^{2}+\epsilon_{2}\left(\frac{a M_{2}}{\pi\left(\bar{k}_{3}+T_{3}-T_{2}\right)}\right)^{2} \\
& +\epsilon_{3} g_{M_{3}}\left(\frac{\bar{k}_{3}}{a}\right) \\
< & \delta_{3}
\end{aligned}
$$

where we used (32) in the last inequality.

For $t_{3}^{*}=\left(\bar{k}_{3}+T_{3}+1\right) / a$, we have

$$
\begin{aligned}
\left(H_{\delta_{3}}^{q_{1}} \psi_{3}\right)\left(t_{3}^{*}\right)= & \sum_{\substack{k=-\infty \\
\left|\psi_{3}(k / a)\right| \geq \delta_{3}}}^{\infty} \psi_{2}\left(\frac{k}{a}\right)\left(H q_{1}\right)\left(t-\frac{k}{a}\right) \\
= & \sum_{\substack{k=-\infty \\
\left|\psi_{3}(k / a)\right| \geq \delta_{3}}}^{\infty} \psi_{3}\left(\frac{k}{a}\right) \frac{1}{\pi\left(t_{3}^{*}-\frac{k}{a}\right)} \\
& -\sum_{\substack{k=-\infty \\
\left|\psi_{3}(k / a)\right| \geq \delta_{3}}}^{\infty} \psi_{3}\left(\frac{k}{a}\right) r\left(t_{3}^{*}-\frac{k}{a}\right) .
\end{aligned}
$$


Since $\max \left\{k: \psi_{3}(k / a) \geq \delta_{3}\right\}<t_{3}^{*} a$ according to (36), every summand of the first sum on the right-hand side of (37) is positive, and therefore, leaving out some summands only reduces the sum. Using $\epsilon_{3} g_{M_{3}}(t) \leq \psi_{3}(t), t \in \mathbb{R}$, we obtain

$$
\begin{aligned}
\left(H_{\delta_{3}}^{q_{1}} \psi_{3}\right)\left(t_{3}^{*}\right) \geq & \sum_{\left|k-T_{3}\right|<\bar{k}_{3}} \epsilon_{3} g_{M_{3}}\left(\frac{k}{a}\right) \frac{1}{\pi\left(t_{3}^{*}-\frac{k}{a}\right)} \\
& -\sum_{|k|<\bar{k}_{3}+T_{3}}\left|\psi_{3}\left(\frac{k}{a}\right) r\left(t_{3}^{*}-\frac{k}{a}\right)\right| \\
\geq & \frac{a \delta_{3}}{\pi} \sum_{\left|k-T_{3}\right|<\bar{k}_{3}} \frac{1}{\bar{k}_{3}+T_{3}+1-k} \\
& -\sum_{|k|<\bar{k}_{3}+T_{3}}\left|\psi_{3}\left(\frac{k}{a}\right) r\left(t_{3}^{*}-\frac{k}{a}\right)\right| .
\end{aligned}
$$

Since

$$
\sum_{|k|<\bar{k}_{3}+T_{3}}\left|\psi_{3}\left(\frac{k}{a}\right) r\left(t_{3}^{*}-\frac{k}{a}\right)\right|<\left(1+\epsilon_{2}+\epsilon_{3}\right) a^{2}
$$

by Lemma 2, it follows that

$$
\begin{aligned}
\left(H_{\delta_{3}}^{q_{1}} \psi_{3}\right)\left(t_{3}^{*}\right)> & \frac{a \delta_{3}}{\pi} \sum_{\left|k-T_{3}\right|<\bar{k}_{3}} \frac{1}{\bar{k}_{3}+T_{3}+1-k} \\
& -\left(1+\epsilon_{2}+\epsilon_{3}\right) a^{2} \\
\geq & \frac{a \delta_{3}}{\pi} \log \left(\bar{k}_{3}\right)-\left(1+\epsilon_{2}+\epsilon_{3}\right) a^{2} \\
\geq & 8-\left(1+\epsilon_{2}+\epsilon_{3}\right) a^{2}
\end{aligned}
$$

where we used (31) in the last line.

Now suppose that we have already constructed functions $\psi_{1}, \ldots, \psi_{r}$ and numbers $\delta_{1}, \ldots, \delta_{r}, \epsilon_{1}, \ldots, \epsilon_{r}$, and $T_{1}, \ldots, T_{r}$, where we set $T_{1}=0$ for convenience, and $C_{1}^{(1)}, \ldots, C_{1}^{(r-1)}, C_{2}^{(1)}, \ldots, C_{2}^{(r-2)}, \ldots, C_{r-1}^{(1)}, r \in \mathbb{N}$. Then, we set for $1 \leq n \leq r$

$$
C_{n}^{(r+1-n)}=\frac{1}{2}\left(\delta_{n}-\psi_{n}\left(\frac{\bar{k}_{n}+T_{n}}{a}\right)-\sum_{l=n+1}^{r} \epsilon_{l}\right)
$$

and choose some $\epsilon_{r+1}$ satisfying

$$
0<\epsilon_{r+1}<\frac{1}{3} \min _{1 \leq n \leq r} C_{n}^{(r+1-n)}
$$

and some $\delta_{r+1}$ satisfying $1 / \pi^{2} \epsilon_{r+1}<\delta_{r+1}<\epsilon_{r+1}$. Furthermore, let $M_{r+1}>M_{r}$ be the smallest natural number for which

$$
\frac{a \delta_{r+1}}{\pi} \log \left(x_{\eta_{r+1}} a M_{r+1}\right) \geq 2^{r+1}
$$

where $\eta_{r+1}=\delta_{r+1} / \epsilon_{r+1}$. Then, we set

$$
\psi_{r+1}(t)=\psi_{r}(t)+\epsilon_{r+1} g_{M_{r+1}}\left(t-\frac{T_{r+1}}{a}\right)
$$

where $T_{r+1}$ is the smallest natural number for which

$\sum_{l=1}^{r} \epsilon_{l}\left(\frac{a M_{l}}{\pi\left(T_{r+1}+\bar{k}_{r+1}-T_{l}\right)}\right)^{2}<\delta_{r+1}-\epsilon_{r+1} g_{M_{r+1}}\left(\frac{\bar{k}_{r+1}}{a}\right)$.
Because of the special construction of $\psi_{r+1}$, we have for arbitrary $1 \leq l \leq r+1$ that

$$
\psi_{r+1}\left(\frac{k}{a}\right)<\delta_{l}
$$

for all $k \geq \bar{k}_{l}+T_{l}$ and consequently for all $k \geq t_{l}^{*} a$. It follows for $1 \leq l \leq r+1$ and $t_{l}^{*}=\left(\bar{k}_{l}+T_{l}+1\right) / a$ that

$$
\begin{aligned}
\left(H_{\delta_{l}}^{q_{1}} \psi_{r+1}\right)\left(t_{l}^{*}\right)= & \sum_{\substack{k=-\infty \\
\left|\psi_{r+1}(k / a)\right| \geq \delta_{l}}}^{\infty} \psi_{r+1}\left(\frac{k}{a}\right)\left(H q_{1}\right)\left(t-\frac{k}{a}\right) \\
= & \sum_{\substack{k=-\infty \\
\left|\psi_{r+1}(k / a)\right| \geq \delta_{l}}}^{\infty} \psi_{r+1}\left(\frac{k}{a}\right) \frac{1}{\pi\left(t_{l}^{*}-\frac{k}{a}\right)} \\
& -\sum_{\substack{k=-\infty \\
\left|\psi_{r+1}(k / a)\right| \geq \delta_{l}}}^{\infty} \psi_{r+1}\left(\frac{k}{a}\right) r\left(t_{l}^{*}-\frac{k}{a}\right) .
\end{aligned}
$$

Since $\max \left\{k: \psi_{r+1}(k / a) \geq \delta_{l}\right\}<t_{l}^{*} a$ according to (36), every summand of the first sum on the right-hand side of (41) is positive, and therefore, leaving out some summands only reduces the sum. Using $\epsilon_{l} g_{M_{l}}(t) \leq \psi_{r+1}(t), t \in \mathbb{R}, 1 \leq l \leq r+1$, we obtain

$$
\begin{aligned}
\left(H_{\delta_{l}}^{q_{1}} \psi_{r+1}\right)\left(t_{l}^{*}\right) \geq & \sum_{\left|k-T_{l}\right|<\bar{k}_{l}} \epsilon_{l} g_{M_{l}}\left(\frac{k}{a}\right) \frac{1}{\pi\left(t_{l}^{*}-\frac{k}{a}\right)} \\
& -\sum_{|k|<\bar{k}_{l}+T_{l}}\left|\psi_{r+1}\left(\frac{k}{a}\right) r\left(t_{l}^{*}-\frac{k}{a}\right)\right| \\
\geq & \frac{a \delta_{l}}{\pi} \sum_{\left|k-T_{l}\right|<\bar{k}_{l}} \frac{1}{\bar{k}_{l}+T_{l}+1-k} \\
& -\sum_{|k|<\bar{k}_{l}+T_{l}}\left|\psi_{r+1}\left(\frac{k}{a}\right) r\left(t_{l}^{*}-\frac{k}{a}\right)\right| .
\end{aligned}
$$

Since

$$
\sum_{|k|<\bar{k}_{l}+T_{l}}\left|\psi_{r+1}\left(\frac{k}{a}\right) r\left(t_{l}^{*}-\frac{k}{a}\right)\right|<a^{2} \sum_{k=1}^{r+1} \epsilon_{k}
$$

by Lemma 2 , it follows that

$$
\begin{aligned}
\left(H_{\delta_{l}}^{q_{1}} \psi_{r+1}\right)\left(t_{l}^{*}\right) & >\frac{a \delta_{l}}{\pi} \log \left(\bar{k}_{l}\right)-a^{2} \sum_{k=1}^{r+1} \epsilon_{k} \\
& \geq 2^{l}-a^{2} \sum_{k=1}^{r+1} \epsilon_{k} .
\end{aligned}
$$

Note that according to our construction of $\epsilon_{l}, l \in \mathbb{N}$, we have

$$
\sum_{l=1}^{\infty} \epsilon_{l}<1+\delta_{1}-\psi_{1}\left(\bar{k}_{1}\right)<2
$$

which implies that

$$
\left(H_{\delta_{l}}^{q_{1}} \psi_{r+1}\right)\left(t_{l}^{*}\right)>2^{l}-2 a^{2}
$$

for all $1 \leq l \leq r+1$. 
The sequence of functions $\left\{\psi_{k}\right\}_{k \in \mathbb{N}}$, where

$$
\psi_{k}(t)=\sum_{l=1}^{k} \epsilon_{l} g_{M_{l}}\left(t-\frac{T_{l}}{a}\right)
$$

with $\left\|g_{M_{l}}\right\|_{\mathcal{P W}_{\pi}^{1}}=1$ for all $l \in \mathbb{N}$, converges to a function

$$
\Phi_{1}(t)=\sum_{l=1}^{\infty} \epsilon_{l} g_{M_{l}}\left(t-\frac{T_{l}}{a}\right)
$$

which is in $\mathcal{P} \mathcal{W}_{\pi}^{1}$, because

$$
\left\|\Phi_{1}\right\|_{\mathcal{P} \mathcal{W}_{\pi}^{1}} \leq \sum_{l=1}^{\infty}\left|\epsilon_{l}\right|\left\|g_{M_{l}}\right\|_{\mathcal{P} \mathcal{W}_{\pi}^{1}}<2 .
$$

Since (40) is valid for all $r \in \mathbb{N}$, we have for all $k \geq \bar{k}_{l}+T_{l}$, we obtain $l \in \mathbb{N}$

$$
0 \leq \Phi_{1}\left(\frac{k}{a}\right)<\delta_{l},
$$

and therefore, it follows that

$$
\begin{aligned}
\left(H_{\delta_{l}}^{q_{1}} \Phi_{1}\right)\left(t_{l}^{*}\right)= & \sum_{\substack{k=-\infty \\
\left|\Phi_{1}(k / a)\right| \geq \delta_{l}}}^{\infty} \Phi_{1}\left(\frac{k}{a}\right)\left(H q_{1}\right)\left(t_{l}^{*}-\frac{k}{a}\right) \\
= & \sum_{\substack{k=-\infty \\
\left|\Phi_{1}(k / a)\right| \geq \delta_{l}}}^{\infty} \Phi_{1}\left(\frac{k}{a}\right) \frac{1}{\pi\left(t_{l}^{*}-\frac{k}{a}\right)} \\
& -\sum_{\substack{k=-\infty \\
\left|\Phi_{1}(k / a)\right| \geq \delta_{l}}}^{\infty} \Phi_{1}\left(\frac{k}{a}\right) r\left(t_{l}^{*}-\frac{k}{a}\right) \\
\geq & \sum_{\left|k-T_{l}\right|<\bar{k}_{l}} \epsilon_{l} g_{M_{l}}\left(\frac{k}{a}\right) \frac{1}{\pi\left(t_{l}^{*}-\frac{k}{a}\right)} \\
& -\sum_{|k|<\bar{k}_{l}+T_{l}}\left|\Phi_{1}\left(\frac{k}{a}\right) r\left(t_{l}^{*}-\frac{k}{a}\right)\right| \\
> & \frac{a \delta_{l}}{\pi} \log \left(\bar{k}_{l}\right)-2 a^{2} \\
> & 2^{l}-2 a^{2} .
\end{aligned}
$$

This implies

$$
\left\|H_{\delta_{l}}^{q_{1}} \Phi_{1}\right\|_{\infty}>2^{l}-2 a^{2}
$$

for all $l \in \mathbb{N}$ and consequently

$$
\limsup _{\delta \rightarrow 0}\left\|H_{\delta}^{q_{1}} \Phi_{1}\right\|_{\infty}=\infty .
$$

Finally, we can divide $\Phi_{1}$ by its norm to obtain the signal

$$
f_{1}=\frac{\Phi_{1}}{\left\|\Phi_{1}\right\|_{\mathcal{P W}}^{1}} .
$$

Since

$$
\begin{aligned}
\left\|H_{\delta}^{q_{1}} f_{1}\right\|_{\infty} & \left\|\sum_{\substack{k=-\infty \\
\left|f_{1}(k / a)\right| \geq \delta}}^{\infty} f_{1}\left(\frac{k}{a}\right)\left(H q_{1}\right)\left(\cdot-\frac{k}{a}\right)\right\|_{\infty} \\
= & \frac{1}{\left\|\Phi_{1}\right\|_{\mathcal{P} \mathcal{W}_{\pi}^{1}}} \\
& \times\left\|\sum_{\substack{k=-\infty \\
\left|\Phi_{1}(k / a)\right| \geq \delta\left\|\Phi_{1}\right\|_{\mathcal{P} \mathcal{W}_{\pi}^{1}}}}^{\infty} \Phi_{1}\left(\frac{k}{a}\right)\left(H q_{1}\right)\left(\cdot-\frac{k}{a}\right)\right\|_{\infty} \\
= & \frac{1}{\left\|\Phi_{1}\right\|_{\mathcal{P} \mathcal{W}_{\pi}^{1}}}\left\|H_{\delta\left\|\Phi_{1}\right\|_{\mathcal{P W}}}^{q_{1}} \Phi_{1}\right\|_{\infty}
\end{aligned}
$$

$$
\begin{aligned}
\limsup _{\delta \rightarrow 0}\left\|H_{\delta}^{q_{1}} f_{1}\right\|_{\infty} & =\frac{1}{\left\|\Phi_{1}\right\|_{\mathcal{P} \mathcal{W}_{\pi}^{1}}} \limsup _{\delta \rightarrow 0}\left\|H_{\delta\left\|\Phi_{1}\right\|_{\mathcal{P} \mathcal{W}_{\pi}^{1}}^{q_{1}}} \Phi_{1}\right\|_{\infty} \\
& =\infty
\end{aligned}
$$

which completes the proof.

\section{NuMERICAL EXAMPLE}

In this section, we illustrate the divergence phenomenon encountered in Theorem 1. Although the proof of Theorem 1 provides an explicit procedure for the construction of the divergence creating signal $f_{1}$, it is numerical problematic to compute this signal, because it is created through an infinite iterative process and the numbers involved in its construction increase rapidly. Therefore, we use a slightly different signal for the simulation.

For the simulation, we use the signal

$$
f_{\mathrm{s}}(t)=\sum_{l=7}^{\infty} \epsilon_{l} g_{M_{l}}\left(t-\frac{T_{l}}{a}\right)
$$

with $\epsilon_{l}=1 /(\sigma l)^{2}$ and $M_{l}=2^{\left((\sigma l)^{3}\right)}$, where $\sigma=1 / 5$ was introduced only for numerical purposes to reduce the magnitude of the numbers in the simulation. The functions

$$
g_{M}(t)=\left(\frac{\sin \left(\frac{\pi t}{M}\right)}{\frac{\pi t}{M}}\right)^{2}, \quad M \in \mathbb{R}, \quad M>0 .
$$

are the same basic elements as in the proof of Theorem 1 . The only difference is that we allow $M$ to be any positive real number here. The translations $T_{l} \in \mathbb{N}$ are chosen such that the basic functions $g_{M_{l}}$ are separated far enough not to disturb each other. In particular, we require that for all $l \geq 7$

$$
f_{\mathrm{s}}(k / a)<\delta_{l}, \quad \text { for all }|k| \geq \bar{k}_{l}+T_{l}
$$


TABLE I

LOWER BOUND $\left(H_{\delta_{l}}^{q_{1}} \epsilon_{l} g_{M_{l}}\right)\left(\left(\bar{k}_{l}+1\right) / a\right)$ FOR DIFFERENT VALUES OF $\delta_{l}$

\begin{tabular}{l|lllllllll}
$l$ & 7 & 8 & 9 & 10 & 11 & 12 & 13 & 14 & 15 \\
$\delta_{l}$ & 0.464 & 0.356 & 0.281 & 0.228 & 0.188 & 0.158 & 0.135 & 0.116 & 0.101 \\
$\left(H_{\delta_{l}}^{q_{1}} \epsilon_{l} g_{M_{l}}\right)\left(\left(\bar{k}_{l}+1\right) / a\right)$ & 0.587 & 0.614 & 0.696 & 0.779 & 0.863 & 0.947 & 1.030 & 1.112 & 1.194
\end{tabular}

where

$$
\delta_{l}=\frac{\epsilon_{l}}{\pi^{2}}+\frac{9}{10}\left(\epsilon_{l}-\frac{\epsilon_{l}}{\pi^{2}}\right)
$$

and $\bar{k}_{l}=k_{\delta_{l} / \epsilon_{l}}\left(M_{l}\right)$. This can be achieved using the same procedure as in the proof of Theorem 1 . We will see that the specific values of the translations $T_{l}$ do not matter for the numerical simulation.

Next, we show numerically what we have proved in Theorem 1: the peak value of the approximation $\left\|H_{\delta_{l}}^{q_{1}} f_{\mathrm{s}}\right\|_{\infty}$ increases as $l$ is increased, i.e., as the threshold $\delta_{l}$ is decreased. Since $\left\|H_{\delta_{l}}^{q_{1}} f_{\mathrm{s}}\right\|_{\infty} \geq\left(H_{\delta_{l}}^{q_{1}} f_{\mathrm{s}}\right)\left(t_{l}^{*}\right)$, for $t_{l}^{*}=\left(\bar{k}_{l}+T_{l}+1\right) / a$, it is sufficient to evaluate $\left(H_{\delta_{l}}^{q_{1}} f_{\mathrm{s}}\right)\left(t_{l}^{*}\right)$ numerically. However, the numerical evaluation can be further simplified, because

$$
\begin{aligned}
\left(H_{\delta_{l}}^{q_{1}} f_{\mathrm{s}}\right)\left(t_{l}^{*}\right) & =\sum_{\substack{k=-\infty \\
\left|f_{\mathrm{s}}(k / a)\right| \geq \delta_{l}}}^{\infty} f_{\mathrm{s}}\left(\frac{k}{a}\right)\left(H q_{1}\right)\left(t_{l}^{*}-\frac{k}{a}\right) \\
& =\sum_{\substack{|k|<\bar{k}_{l}+T_{l} \\
\left|f_{\mathrm{s}}(k / a)\right| \geq \delta_{l}}} f_{\mathrm{s}}\left(\frac{k}{a}\right)\left(H q_{1}\right)\left(t_{l}^{*}-\frac{k}{a}\right)
\end{aligned}
$$

according to (43). Since $\left(H q_{1}\right)(t)>0$ for $t>0$, which follows directly from (15), and $g_{M_{l}}(t)>0$ for all $l \in \mathbb{N}, t \in \mathbb{R}$, all summands in (44) are positive and leaving out some summands only reduces the sum. Thus, we obtain

$$
\begin{aligned}
& \left(H_{\delta_{l}}^{q_{1}} f_{\mathrm{s}}\right)\left(t_{l}^{*}\right) \geq \sum_{\substack{\left|k-T_{1}\right|<\bar{k}_{l} \\
\left|f_{\mathrm{s}}(k / a)\right| \geq \delta_{l}}} f_{\mathrm{s}}\left(\frac{k}{a}\right)\left(H q_{1}\right)\left(t_{l}^{*}-\frac{k}{a}\right) \\
& \geq \sum_{\left|k-T_{l}\right|<\bar{k}_{l}} \epsilon_{l} g_{M_{l}}\left(\frac{k-T_{l}}{a}\right)\left(H q_{1}\right)\left(t_{l}^{*}-\frac{k}{a}\right) \\
& =\sum_{|k|<\bar{k}_{l}} \epsilon_{l} g_{M_{l}}\left(\frac{k}{a}\right)\left(H q_{1}\right)\left(\frac{\bar{k}_{l}+1}{a}-\frac{k}{a}\right) \\
& =\left(H_{\delta_{l}}^{q_{1}} \epsilon_{l} g_{M_{l}}\right)\left(\frac{\bar{k}_{l}+1}{a}\right) \text {. }
\end{aligned}
$$

show the unbounded increase of the lower bound numerically in a finite number of simulation runs. Nevertheless, Table I shows the increasing trend of the lower bound.

It is interesting to discuss the divergence of $\left\|H_{\delta_{l}}^{q_{1}} f_{\mathrm{s}}\right\|_{\infty}$ a little further and to compare its divergence speed with the divergence speed of the peak value of the Shannon sampling series. In Section II-A, we have seen that there exists a signal $f_{1} \in \mathcal{P} \mathcal{W}_{\pi}^{1}$ such that $\lim \sup _{N \rightarrow \infty}\left\|S_{N} f_{1}\right\|_{\infty}=\infty$, where

$$
\left(S_{N} f\right)(t)=\sum_{k=-N}^{N} f(k) \frac{\sin (\pi(t-k))}{\pi(t-k)}
$$

denotes the finite Shannon sampling series. In [12], it was shown that the divergence speed of $\left\|S_{N} f_{1}\right\|_{\infty}$ is at most of the order $\log (N)$. If we have a look at the construction of the signal $f_{\mathrm{s}}$, we see that the situation is different here. The determining factor for the divergence speed of $\left\|H_{\delta_{l}}^{q_{1}} f_{\mathrm{s}}\right\|_{\infty}$ is the growth rate of the sequence $\left\{M_{l}\right\}_{l \in N}$. However, this rate can be made arbitrarily large. Hence, for any desired divergence speed order, it is possible to construct a signal $f_{2} \in \mathcal{P} \mathcal{W}_{\pi}^{1}$ such that $\left\|H_{\delta_{l}}^{q_{1}} f_{2}\right\|_{\infty}$ diverges with this order.

\section{REFERENCES}

[1] B. F. Logan, Jr., "Signals designed for recovery after clipping-I. Localization of infinite products," AT\&T Bell Lab. Tech. J., vol. 63, no. 2 , pp. 261-285, Feb. 1984.

[2] B. F. Logan, Jr., "Signals designed for recovery after clipping-II. Fourier transform theory of recovery," AT\&T Bell Lab. Tech. J., vol. 63, no. 2, pp. 287-306, Feb. 1984.

[3] B. F. Logan, Jr., "Signals designed for recovery after clipping-III: Generalizations," AT\&T Bell Lab. Tech. J., vol. 63, no. 3, pp. 379-399, Mar. 1984.

[4] H. W. Kuhn and S. Nasar, Eds., The Essential John Nash. Princeton, NJ: Princeton Univ. Press, 2007.

[5] J. W. Milnor, "Games against nature," RAND Corp., Santa Monica, CA, Res. Memorandum, 1951.

[6] K. J. Arrow, "A difficulty in the concept of social welfare," J. Political Econ., vol. 58, no. 4, pp. 328-346, 1950

[7] L. S. Shapley, "A value for n-person games," RAND Corp., 1952, .

[8] J. F. Nash, Jr., "The bargaining problem," Econometrica, vol. 18, no. 2, pp. 155-162, 1950.

[9] J. L. Brown, Jr., "On the error in reconstructing a non-bandlimited function by means of the bandpass sampling theorem," J. Math. Anal. Appl., vol. 18, pp. 75-84, 1967.

[10] P. L. Butzer, W. Splettstößer, and R. L. Stens, "The sampling theorem and linear prediction in signal analysis," Jahresber. d. Dt. Math. Verein., vol. 90, no. 1, pp. 1-70, Jan. 1988.

Hence, $\left(H_{\delta_{l}}^{q_{1}} \epsilon_{l} g_{M_{l}}\right)\left(\left(\bar{k}_{l}+1\right) / a\right)$ is a lower bound for the peak value of the reconstruction $\left\|H_{\delta_{l}}^{q_{1}} f_{\mathrm{s}}\right\|_{\infty}$. In Table I, we see that this lower bound increases as $\delta$ is decreased along the sequence $\delta_{1}, \delta_{2}, \ldots, \delta_{15}$.

Note that $\left(H_{\delta_{l}}^{q_{1}} \epsilon_{l} g_{M_{l}}\right)\left(\left(\bar{k}_{l}+1\right) / a\right)$ is only a lower bound for the peak value of the reconstruction $\left\|H_{\delta_{l}}^{q_{1}} f_{\mathrm{s}}\right\|_{\infty}$. So the increase of this lower bound for a finite number of thresholds does not necessarily imply the increase of $\left\|H_{\delta_{l}}^{q_{1}} f_{\mathrm{s}}\right\|_{\infty}$. However, this numerical example is only meant to illustrate what we have mathematically proven in Section IV. From the proof of Theorem 1, we know that the lower bound $\left(H_{\delta_{l}}^{q_{1}} \epsilon_{l} g_{M_{l}}\right)\left(\left(\bar{k}_{l}+1\right) / a\right)$ increases unboundedly, which in turn implies the unbounded increase of $\left\|H_{\delta_{l}}^{q_{1}} f_{\mathrm{s}}\right\|_{\infty}$ as the threshold tends to zero. Of course, we cannot

[11] P. L. Butzer and R. L. Stens, "Sampling theory for not necessarily bandlimited functions: A historical overview," SIAM Rev., vol. 34, no. 1, pp. 40-53, Mar. 1992.

[12] H. Boche and U. J. Mönich, "There exists no globally uniformly convergent reconstruction for the Paley-Wiener space $\mathcal{P} \mathcal{W}_{\pi}^{1}$ of bandlimited functions sampled at Nyquist rate," IEEE Trans. Signal Process. vol. 56, no. 7, pp. 3170-3179, Jul. 2008.

[13] H. Boche and U. J. Mönich, "Impact of thresholding on signal processing performance with applications," in Proc. 9th Int. Conf. Signal Process., 2008, pp. 2604-2607.

[14] J. L. Brown, Jr., "Analytic signals and product theorems for Hilbert transforms," IEEE Trans. Circuits Syst., vol. 21, no. 6, pp. 790-792, Nov. 1974.

[15] J. L. Brown, Jr., "On the error in reconstructing a non-bandlimited function by means of the bandpass sampling theorem," J. Math. Anal. Appl., vol. 21 , p. 699 , 1968, Erratum. 


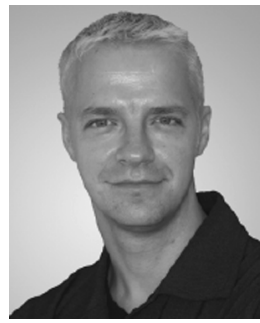

Holger Boche (M'04-SM'07) received the Dipl.-Ing. and Dr.-Ing. degrees in electrical engineering from the Technische Universität Dresden, Dresden, Germany, in 1990 and 1994, respectively. He graduated in mathematics from the Technische Universität Dresden, in 1992 and received the Dr.rer.nat. degree in pure mathematics from the Technische Universität Berlin, Berlin, Germany, in 1998. From 1994 to 1997 , he did postgraduate studies in mathematics at the Friedrich-Schiller Universität, Jena, Germany.

In 1997, he joined the Heinrich-Hertz-Institut (HHI) für Nachrichtentechnik, Berlin, Germany. Since 2002, he has been Full Professor for Mobile Communication Networks at the Institute for Communications Systems, the Technische Universität Berlin. In 2003, he became the Director of the Fraunhofer GermanSino Lab for Mobile Communications, Berlin, Germany and since 2004 he has also been the Director of the Fraunhofer Institute for Telecommunications (HHI), Berlin, Germany. He was visiting professor at the ETH Zurich during winter 2004 and 2006 and at KTH Stockholm during summer 2005.

Prof. Boche received the Research Award "Technische Kommunikation" from the Alcatel SEL Foundation in October 2003, the "Innovation Award" from the Vodafone Foundation in June 2006, and the Gottfried Wilhelm Leibniz Prize from the Deutsche Forschungsgemeinschaft (German Research Foundation) in 2008. He was corecipient of the 2006 IEEE Signal Processing Society Best Paper Award and recipient of the 2007 IEEE Signal Processing Society Best Paper Award. He is a member of IEEE Signal Processing Society SPCOM and SPTM Technical Committee. He was elected a member of the German Academy of Sciences (Leopoldina) in 2008.

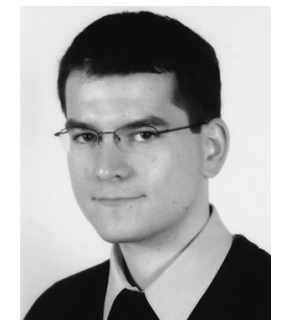

Ullrich J. Mönich (S'06) received the Dipl.-Ing. degree in electrical engineering from the Technische Universität Berlin, Berlin, Germany in 2005, where he is currently working towards the Ph.D. degree.

During winter 2003, he was a visiting researcher at the University of California, Santa Barbara. His research activities comprise sampling theory, ultrawideband mobile communications, and information theory. 